\title{
Presión Osmótica de Soluciones Salinas y Azucaradas: su Influencia en Procesos de Osmosis Inversa en la Industria de Alimentos
}

\author{
Beatriz Cancino, Lila Ulloa y Carolina Astudillo \\ Pontificia Universidad Católica de Valparaíso, Escuela de Alimentos, Waddington 716, \\ Valparaíso-Chile (email: beatriz.cancino@ucv.cl)
}

\begin{abstract}
Resumen
En este trabajo se determinó el efecto de la concentración de las soluciones salinas y azucaradas, presentes comúnmente en la industria de alimentos, en la presión osmótica durante procesos de osmosis inversa. Las soluciones salinas fueron $\mathrm{NaCl}$ y $\mathrm{KCl}$, mientras que las azucaradas fueron $\mathrm{D}$ Fructosa y Lactosa. Las concentraciones estudiadas fueron $0.5,0.8,1.0,1.5,2.0$, y $5.0 \% \mathrm{p} / \mathrm{v}$ de cada una. Se determinó la presión osmótica mediante un procedimiento experimental que involucra determinar la resistencia de la membrana de osmosis inversa (acetato de celulosa) y la resistencia del proceso para cada concentración. Se comparó los resultados de presión osmótica con los obtenidos con las ecuaciones de Van't Hoff y la de Gibbs, concluyendo que ninguno de estos modelos representa adecuadamente el comportamiento de las soluciones de azúcares con concentraciones mayores al $2 \% \mathrm{p} / \mathrm{v}$. Esta divergencia se acentúa en soluciones salinas.
\end{abstract}

\section{Osmotic Pressure of Salt and Sugar Solutions: its Influence on Reverse Osmosis in the Food Industry}

\begin{abstract}
The effect of the concentration of salt and sugar solutions on osmotic pressure during reverse osmosis processes was determined. Salty solutions were $\mathrm{NaCl}$ and $\mathrm{KCl}$, while sweet solutions were D-Fructose and Lactose. The studied concentrations were $0.5,0.8,1.0,1.5,2.0,5.0 \% \mathrm{w} / \mathrm{v}$ each. The osmotic pressure was determined by an experimental procedure which involves determining the membrane resistance (cellulose acetate) and the total process resistance for each concentration. Results of osmotic pressure were compared to those predicted by Van't Hoff and Gibbs models, concluding that none of them agrees with the experimental results for sugar concentrations higher than $2.0 \% \mathrm{w} / \mathrm{v}$. This difference between predicted and experimental osmotic pressures is greater in the salt solutions.
\end{abstract}

Keywords: Reverse osmosis, osmotic pressure, salt solutions, sugar solutions, food industry 


\section{INTRODUCCIÓN}

Las soluciones salinas y azucaradas se encuentran en la industria de alimentos tanto en las materias primas, su procesamiento y en los residuos generados (Hinková et al., 2000; Hinková et al., 2002; Cuartas et al., 2004; Cuartas-Uribe et al., 2007). Por ello se requieren de alternativas de procesos para concentrarlas, o para separarlas, siendo interesante estudiar el proceso de osmosis inversa, debido a que permite la recuperación de compuestos, mediante la permeación de agua y concentrando los solutos, como sales o azúcares (Cheryan, 1998), como así también la recuperación de aguas en la industria de alimentos (Galambos et al., 2004).

Por ejemplo, la industria láctea genera una gran cantidad de residuos líquidos, en el rango de 0,2 a 10 litros por litro de leche procesada (Balannec et al., 2005). La osmosis inversa ha sido propuesta para el tratamiento de éste tipo de efluentes, ya que este proceso genera como permeado agua apta para su reciclo dentro de la misma planta (Balannec et al., 2005; Vourch et al., 2005). En otros casos se utiliza para la desmineralización de aguas (Manth et al., 1998; Čuda et al., 2006).

En la industria azucarera, el proceso de osmosis inversa es considerado una atractiva alternativa de bajo consumo energético en reemplazo de los sistemas clásicos de evaporación usados en la concentración de azúcares (Madaeni y Zereshki, 2006; Madaeni y Zereshki, 2008; Hinková et al., 2002). En general, los procesos de membrana son caracterizados por su bajo consumo energético específico (Mirza, 2008). La osmosis inversa ha sido utilizada para la concentración de jugo de uva para la industria vitivinícola (Rektor et al., 2006) y concentración de otros jugos de frutas como naranjas (Silva et al., 1998) y frutas exóticas (Gomes et al., 2005; Cianci et al., 2005).

Para poder dimensionar un proceso de osmosis inversa, lo primero que debe conocerse es el comportamiento de la membrana, es decir el flux de permeado obtenido para cada presión transmembrana aplicada. Este comportamiento puede ser modelado con algunas ecuaciones disponibles en la literatura (Cheryan, 1998; Mulder ,1996; Fariñas, 1999). Para esto es necesario conocer la presión osmótica de la solución que se procesará, la cual dependerá de la concentración y temperatura, así como la resistencia que ejerce la membrana al paso del flux del agua. En relación a esto, existe en la literatura muchos datos en sistemas de osmosis inversa, con membranas sintéticas, sin embargo, sólo se presentan algunos resultados a una presión transmembrana dada (Ludwing et al., 2002), haciendo imposible la determinación de los parámetros operacionales del sistema. Esto dificulta la estimación del ensuciamiento de la membrana, así como de la energía requerida para lograr la separación. Tampoco se puede conocer de antemano el efecto que tendrá el incremento de la concentración de la solución durante un proceso de concentración por osmosis inversa, sobre la presión osmótica de la solución, a menos que se realicen pruebas experimentales específicas para su determinación.

El objetivo de este trabajo es determinar, la presión osmótica de soluciones de azúcar y sales, durante experiencias de osmosis inversa. Además, analizar el efecto que presenta la concentración y la naturaleza del soluto sobre la presión osmótica, el ensuciamiento de la membrana y el requerimiento energético del proceso. Basados en los resultados obtenidos en este trabajo se pudo comprobar que los modelos de Van't Hoff y Gibbs no son adecuados para la estimación de la presión osmótica, validando la contribución de este trabajo para la determinación de la presión osmótica.

\section{MATERIALES Y MÉTODOS}

Materiales

Las soluciones salinas estudiadas fueron: cloruro de sodio y cloruro de potasio; y las soluciones azucaradas: D- Fructosa y Lactosa. Todos los reactivos fueron de calidad p.a. sobre un $99 \%$ de pureza. Las concentraciones estudiadas para cada uno de estos compuestos fueron: $0.5 ; 0.8 ; 1.0$; $1.5 ; 2.0$ y $5 \% \mathrm{p} / \mathrm{v}$. El agua destilada para la preparación de las soluciones presentó una conductividad en un rango de 22 a $50 \mu \mathrm{S} / \mathrm{cm}$.

Se utilizó una membrana plana de acetato de celulosa, tipo W/D 16/96 1/3 Hydraunautics, con un área de filtración de $0.0101795 \mathrm{~m}^{2}$. Se trabajó con una celda plana de acero inoxidable, de alta 
presión, construida y diseñada por GKSS Research Centre (Geesthacht, Alemania) y una bomba de alta presión Speck $\mathrm{GmbH}$ con un rango entre 0 y 140 bar. Un esquema del equipo experimental se muestra en la Fig 1. El equipo fue operado bajo la modalidad de recirculación total, para simular un proceso continuo. La temperatura de las experiencias fue mantenida constante a $20 \pm 2^{\circ} \mathrm{C}$

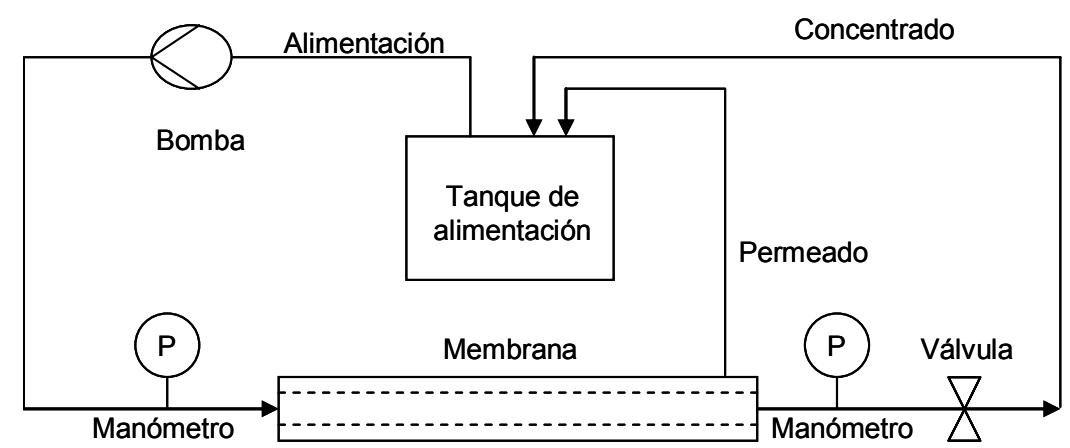

Fig. 1: Esquema del sistema utilizado para la determinación del flux de permeado para las diferentes presiones transmembrana utilizadas.

\section{Métodos}

Para cada soluto y concentración se generaron las curvas experimentales de presión transmembrana y flux de permeado (cuociente entre el flujo de permeado y el área de la membrana). Los datos obtenidos fueron ajustados utilizando el modelo de la presión osmótica (Cheryan, 1998). Este asume que la fuerza motriz para que ocurra la permeación es la diferencia de potencial químico entre dos soluciones. La ecuación de transporte escrita en términos de flux de permeado $(\mathrm{J})$ es la siguiente (Hoek et al., 2002; Cheryan, 1998):

$$
\mathrm{J}=\frac{\mathrm{P}_{\mathrm{T}}-\Delta \Pi}{\mu\left(\mathrm{R}_{\mathrm{M}}+\mathrm{R}_{\mathrm{F}}+\mathrm{R}_{\mathrm{CP}}\right)}
$$

La ecuación 1 muestra el comportamiento del Flux $(\mathrm{J})$ al variar la presión transmembrana $\left(\mathrm{P}_{\mathrm{T}}\right.$, definida como el promedio entre la presión de alimentación y la presión del concentrado), cuando existe un efecto importante de la presión osmótica $(\Delta \Pi)$ de la solución. La resistencia que se opone a la permeación del agua, está dada por la resistencia de la membrana $\left(R_{M}\right)$, la del ensuciamiento reversible o concentración polarización $\left(R_{C P}\right)$ y la del ensuciamiento irreversible $\left(R_{F}\right)$ también conocida como fouling de la membrana. Además se aprecia un efecto explicito de la viscosidad del permeado $(\mu)$ en el flux del permeado (Cheryan, 1998; Mulder, 1996; Fariñas, 1999; Hoek et al., 2002).

La determinación de la resistencia total, puede ser calculada a partir de la pendiente de la recta obtenida de la linealización de los datos experimentales $\left(P_{T} \mathrm{v} / \mathrm{s} \mathrm{J}\right)$. Si se desea determinar la resistencia de la membrana, se repite el mismo protocolo, pero haciendo recircular agua destilada. La diferencia entre la Resistencia Total y la Resistencia de la Membrana entrega información en relación a si existió ensuciamiento producto de la solución empleada. De existir ensuciamiento, la resistencia total para esta solución sería mayor a la del agua pura. En forma cuantitativa, cualquier cambio en la pendiente de la curva $\mathrm{P}_{\mathrm{T}} \mathrm{v} / \mathrm{s} \mathrm{J}$ obtenida con las soluciones, en relación a la del agua pura, será consecuencia del ensuciamiento de la membrana.

La presión osmótica se calculó para cada condición de concentración y para cada soluto, a partir del modelo lineal obtenido de las curvas $P_{\mathrm{T}} \mathrm{v} / \mathrm{s} \mathrm{J}$, empleando la ecuación 1. La presión osmótica se evaluó como la presión transmembrana mínima requerida para que exista algún flux de permeado, es decir $\mathrm{J}=0$, para el modelo.

Los modelos de Van't Hoff (ecuación 2) y el Modelo de Gibbs (ecuación 3) fueron empleados para la estimación de presiones osmóticas de las soluciones (Valentas et al., 1997; Cheryan, 1998; Mulder, 1996; Hoek et al., 2002). En estos modelos $\Pi$ es la presión osmótica de la solución (Pa); $R$ la constante de los gases $(0.08206 \mathrm{~atm} \cdot \mathrm{L} /(\mathrm{mol} \cdot \mathrm{K})) ; \mathrm{X}_{\mathrm{m}}$ la fracción molar de agua; $n$ la concentración 
molar del soluto $\left(\mathrm{mol} / \mathrm{m}^{3}\right)$; $\mathrm{T}$ la temperatura absoluta de la solución $(\mathrm{K})$ y $\mathrm{V}_{\mathrm{m}}$ el volumen molar del solvente en la solución $\left(\mathrm{m}^{3} / \mathrm{mol}\right)$.

$$
\begin{aligned}
& \Pi=\mathrm{n} \cdot \mathrm{R} \cdot \mathrm{T} \\
& \Pi=-\frac{\mathrm{R} \cdot \mathrm{T}}{\mathrm{V}_{\mathrm{m}}} \cdot \ln \mathrm{x}_{\mathrm{m}}
\end{aligned}
$$

\section{RESULTADOS Y DISCUSIÓN}

Todos los experimentos fueron realizados en triplicado, presentando un error experimental menor que un $3.0 \%$. Las correlaciones lineales obtenidas en todos los casos presentaron altos coeficientes de correlación $\left(R^{2}>98\right)$, de acuerdo a la metodología presentada en la sección anterior.

Las Figuras 2, 3, 4 y 5 muestran el efecto de la concentración sobre la presión osmótica para las soluciones de $\mathrm{NaCl}, \mathrm{KCl}$, y Lactosa D- Fructosa, respectivamente. En todas ellas, se muestra la curva realizada sólo con agua destilada la cual pasa cercana al origen. Las curvas con soluto se encuentran desplazadas del origen por efecto de la presión osmótica. Además hay que señalar que para que exista permeación, la presión de operación debe ser superior a la presión osmótica de la solución, como se observa en la Ec.1. En la Fig. 2 aparece un punto cercano a los 40 bar entre los 0 y los $0.01 \mathrm{~L} / \mathrm{m}^{2} \mathrm{~s}$. Esto se debe, a que cuando se trabajó con la solución $5 \% \mathrm{p} / \mathrm{v}$ de $\mathrm{NaCl}$, recién a esa presión transmembrana fue posible obtener permeado. Por lo tanto, es posible suponer que la presión osmótica de dicha solución estaría entre los 35 y 40 bar, de acuerdo al método experimental.

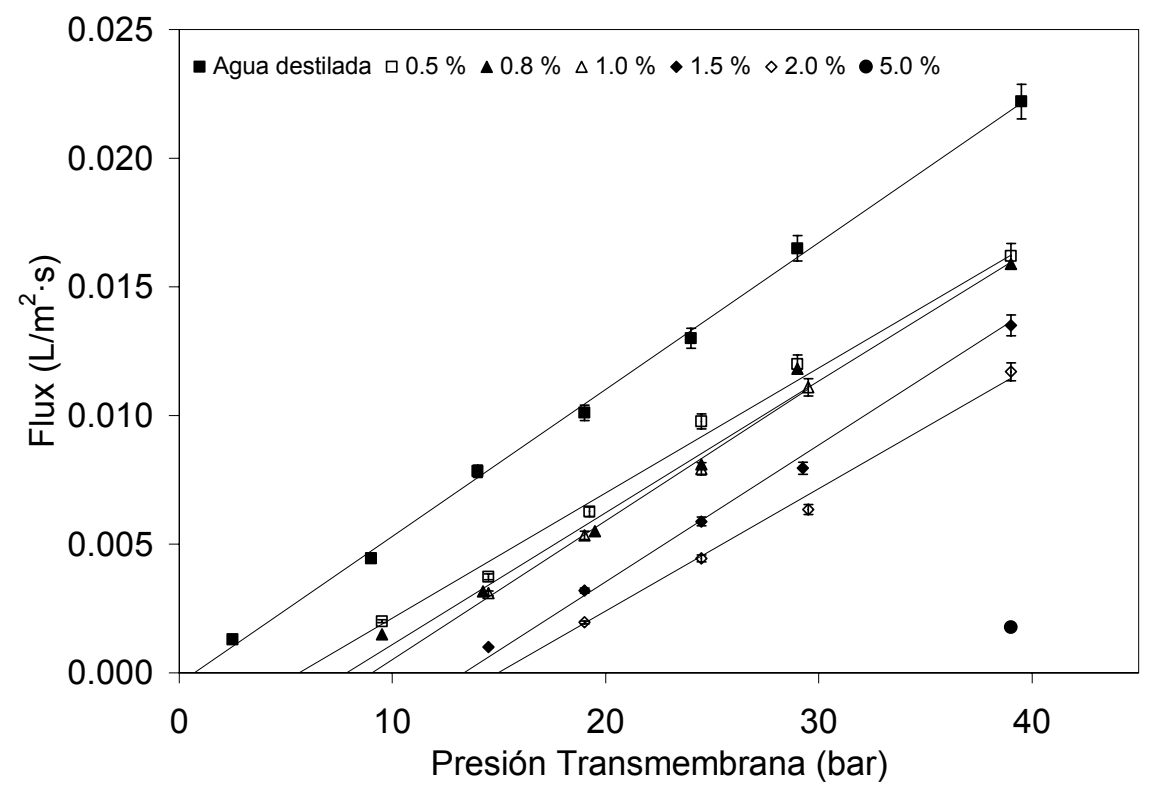

Fig. 2: Variación en el flux de permeado en función de la presion transmembrana para distintas concentraciones de $\mathrm{NaCl}$.

En la Fig. 3 se puede apreciar que para esa misma concentración, la presión osmótica de KCl estaría cercana a los 25 bar. Esto es coherente con el hecho que para iguales concentraciones en \%p/v, la concentración molar de $\mathrm{NaCl}$ es superior (peso molecular $\mathrm{NaCl}=58.44 \mathrm{~g} / \mathrm{mol}$ y $74.56 \mathrm{~g} / \mathrm{mol}$ para $\mathrm{KCl}$ ). Así, el tamaño molecular influye en un mayor gasto de energía para permitir la permeación del líquido a través de la membrana, donde una molécula más pequeña retendrá con mayor energía las moléculas de agua. Además, este fenómeno es consistente con los modelos de Van't Hoff y Gibbs, dado que ambos proponen que la presión osmótica aumenta con la concentración molar de la solución.

En la Fig. 4 se aprecia que soluciones de lactosa comienzan a permear en un rango bastante estrecho de presión transmembrana, lo que significa que la presión osmótica no es una fuerte 
función de la concentración en el rango estudiado. Sin embargo, las pendientes de las curvas varían con la concentración, lo que se relaciona con el ensuciamiento de la membrana (resistencia), que es mayor en la medida que aumenta la concentración de Lactosa.

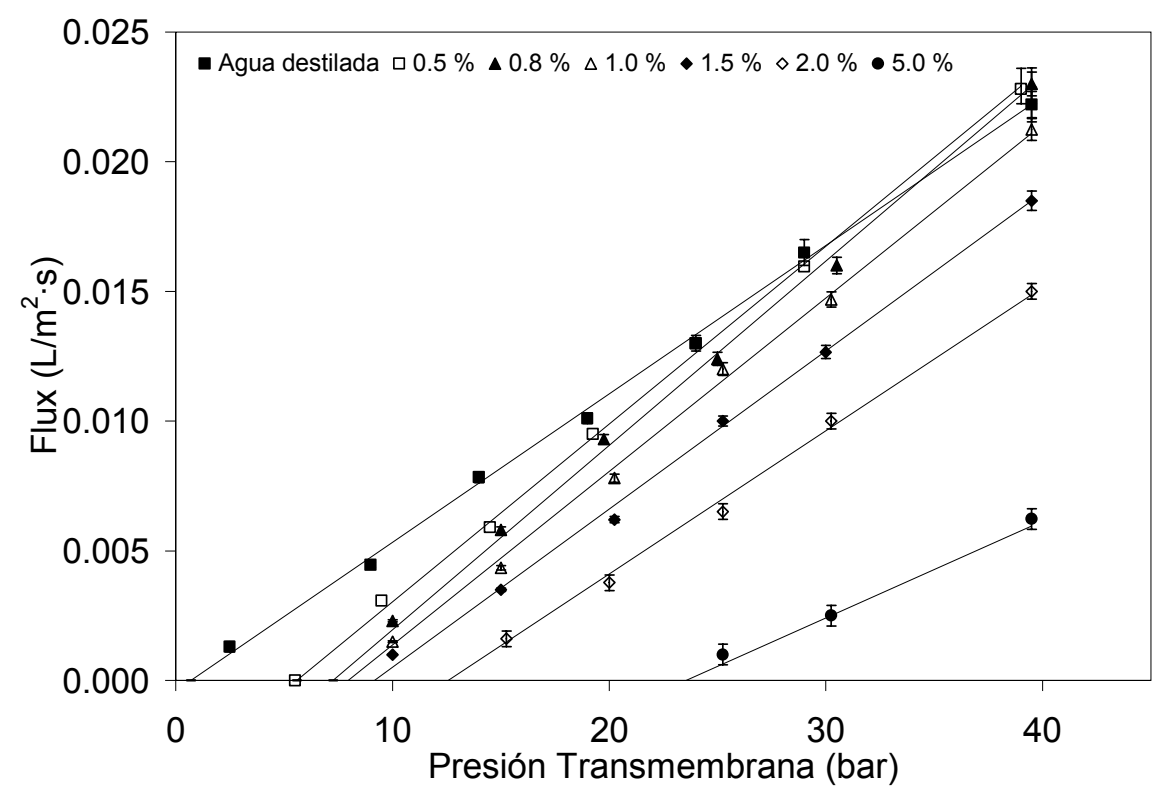

Fig. 3: Variación en el flux de permeado en función de la presion transmembrana para distintas concentraciones de $\mathrm{KCl}$.

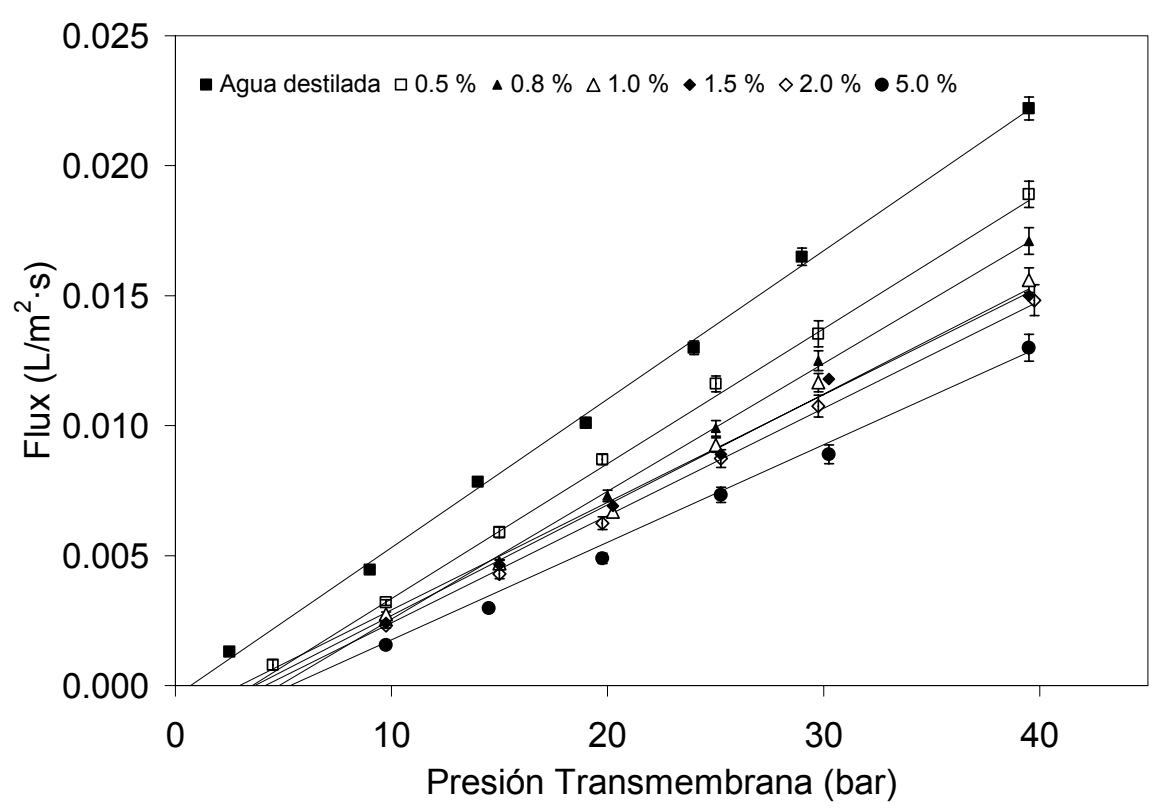

Fig. 4: Variación del Flux de permeado en función de la presión transmembrana para distintas concentraciones de Lactosa.

En la Fig. 5 en cambio, se puede visualizar que la presión transmembrana a la cual comienza la permeación cambia en función con la concentración del azúcar, tal como ocurre con las soluciones de sales. Además, las curvas son prácticamente paralelas, lo que significa que para el rango de concentraciones estudiadas para D-Fructosa, el ensuciamiento de la membrana es independiente de la concentración.

El peso molecular de Lactosa es $342.3 \mathrm{~g} / \mathrm{mol}$ y $180.16 \mathrm{~g} / \mathrm{mol}$ para D-Fructosa. Por lo tanto al igual que para las sales, el efecto del aumento de la presión osmótica con la concentración, se puede deber a que la D-Fructosa es una molecula más pequeña que la lactosa, por lo que interaccionaría 
con mayor energía con las moléculas de agua, y en mayor proporción si se aumenta su concentración. No ocurre lo mismo con la Lactosa debido a que no es tan higroscópica como D-Fructosa y por lo tanto este comportamiento no se ve afectado por el aumento en las concentraciones.

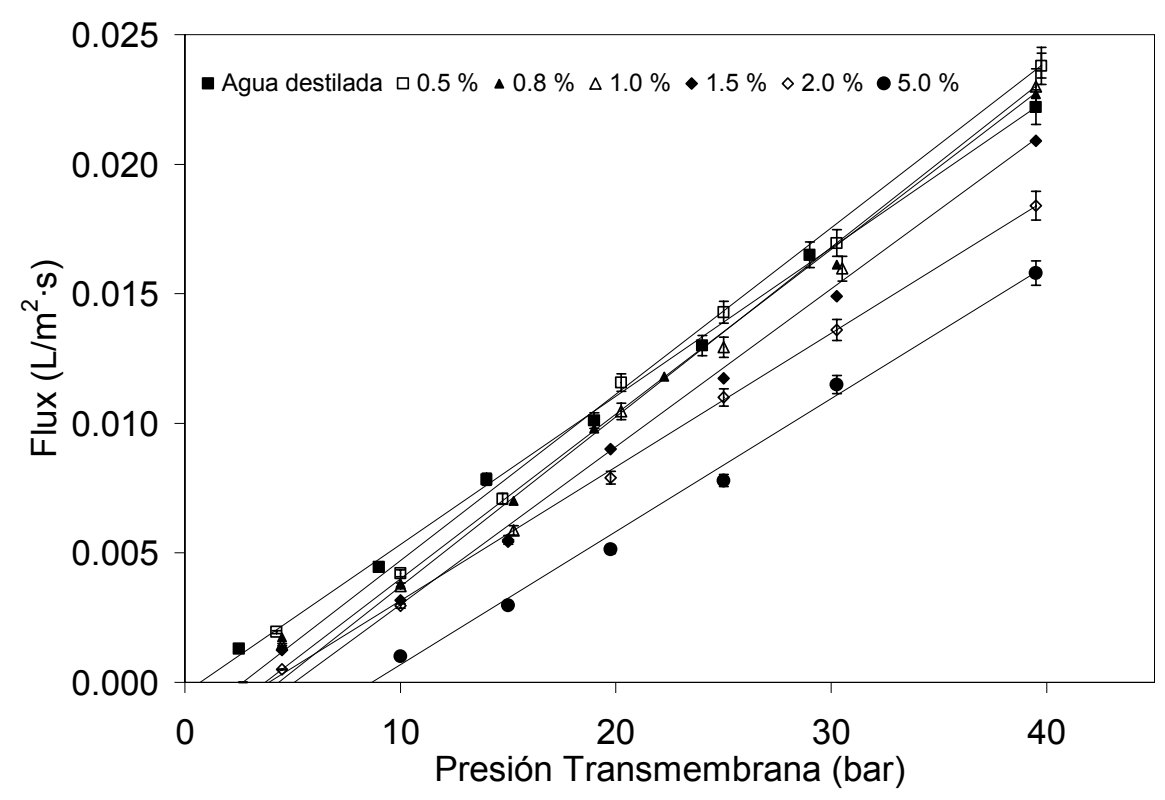

Fig. 5: Variación del Flux de permeado en función de la presión transmembrana para distintas concentraciones de D-Fructosa

La Fig. 6 muestra un resumen comparativo de las diferentes presiones osmóticas obtenidas experimentalmente en función de la concentración para $\mathrm{NaCl}, \mathrm{KCl}$, D-Fructosa y Lactosa.

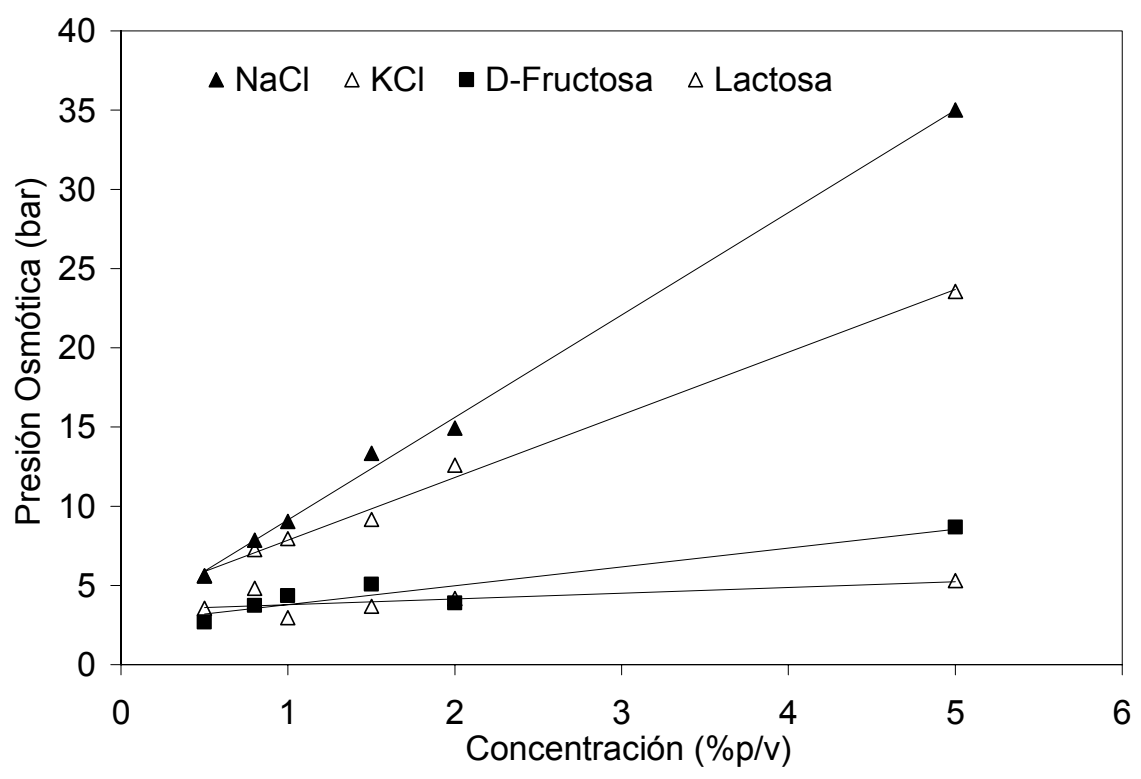

Fig. 6: Presiones osmóticas para las diferentes concentraciones de sales y azucares .

En la Fig. 6, se aprecia que las sales presentan una presión osmótica mayor que las soluciones de azúcares, diferencia que se acentúa al aumentar la concentración. A manera de comparación, Cheryan (1998), entrega datos para presiones osmóticas para Lactosa al $1 \% \mathrm{p} / \mathrm{v}$ es 0.69 bar y al $5 \%$ $\mathrm{p} / \mathrm{v}$ es 3.79 bar, en contraste con lo encontrado experimentalmente en este trabajo que corresponde a 2.97 y 4.16 bar respectivamente. Así también, entrega datos para $\mathrm{NaCl}$ al $1 \%$ de 8.62 bar, valor algo menor al determinado en este trabajo (9.03 bar). Por otra parte Kessler (2002) indica que la presión osmótica $\mathrm{NaCl} 3.5 \%$ p/v es 24.98 bar y Cuartas et al. (2004) determinaron una presión osmótica de 4.6 bar para una mezcla de lactosa al $5 \%$ con sales $\left(\mathrm{NaCl} 0.12 \%\right.$; $\mathrm{KCl} 0.2 \%$ y $\mathrm{MgSO}_{4}$ 
$0.075 \%$ ), ambos valores se encuentran dentro de los rangos determinados en este estudio. De esto se observa que la diferencia entre los valores obtenidos experimentalmente y los entregados por las referencias son mucho mayores para el caso de Lactosa que para $\mathrm{NaCl}$.

La Figuras 7 y 8 , entregan los valores experimentales y los obtenidos de acuerdo a los modelos de Van't Hoff y Gibbs para el $\mathrm{NaCl}$ y el KCl respectivamente. Al observar la Fig. 7 podemos apreciar que a medida que aumenta la concentración de $\mathrm{NaCl}$, la diferencia entre lo que estiman los modelos y los valores experimentales también se incrementa, sobreestimando por lo tanto la energía requerida para dar paso a la permeación en un sistema de osmosis inversa. El mismo fenómeno se observa en la Fig. 8 para el caso de $\mathrm{KCl}$.

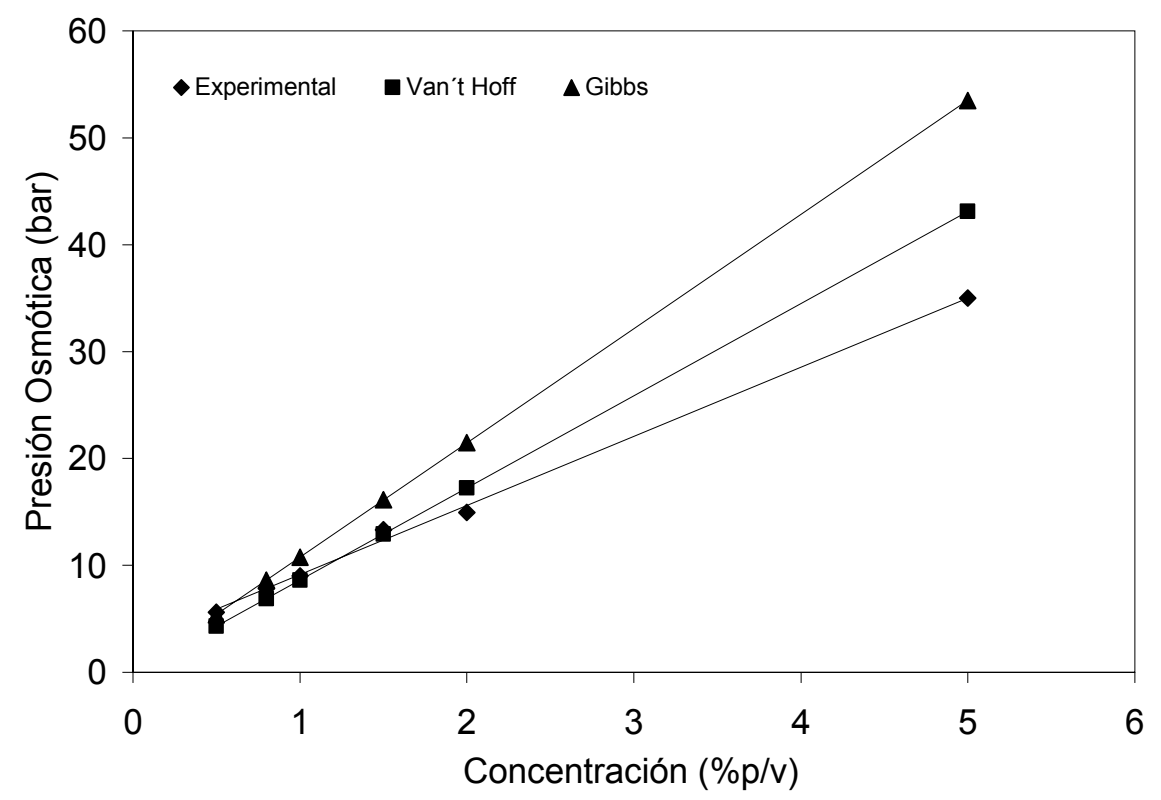

Fig. 7: Valores de Presión osmótica para NaCl según modelos de Van’t Hoff y Gibbs, además de los obtenidos experimentalmente.

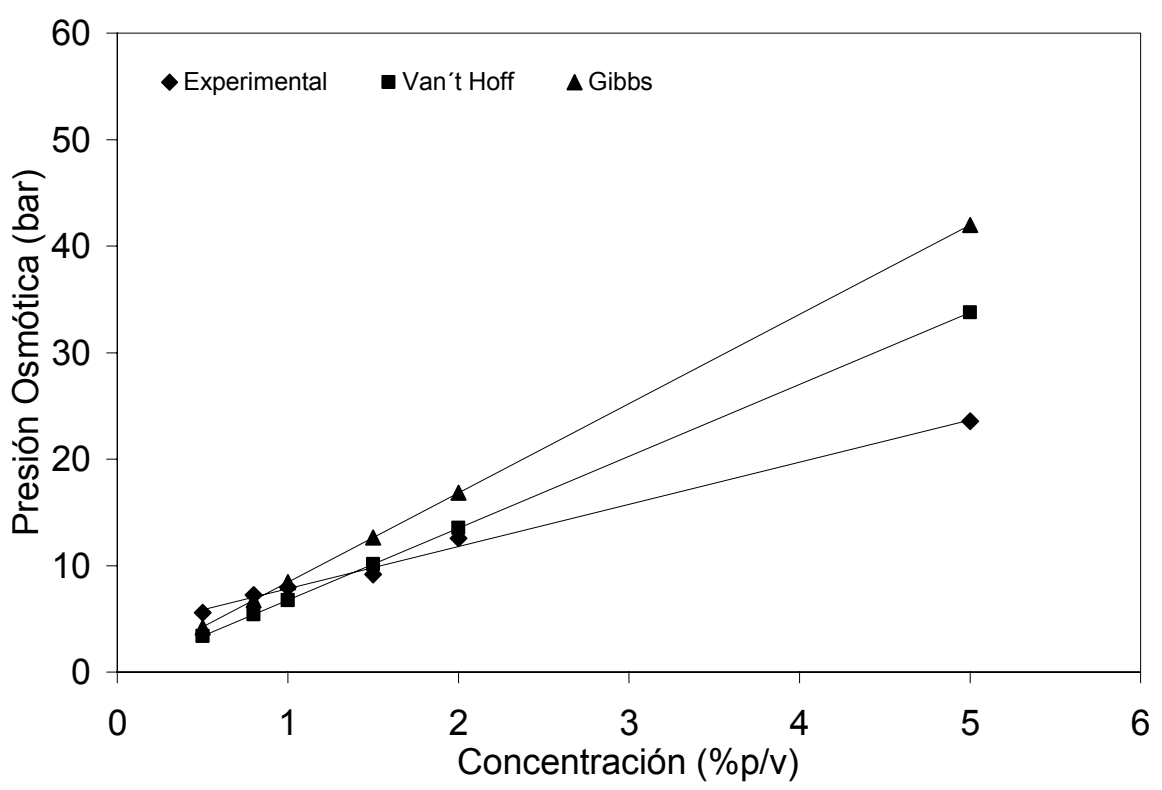

Fig. 8: Valores de Presión osmótica para $\mathrm{KCl}$ según modelos de Van’t Hoff y Gibbs, además de los obtenidos experimentalmente.

En la Tabla 1 se muestran los resultados de presión osmótica para el caso de las soluciones azucaradas, donde se puede observar que las estimaciones realizadas con los modelos, presentan un comportamiento diferente. Para Lactosa, los modelos subestiman la presión osmótica hasta una 
concentración del 2\% para Gibbs y hasta el 5\% para Van't Hoff. Para Fructosa también se observa una subestimación en el valor de presión osmotica hasta una concentración de un 1\%.

Tabla 1: Resultados de la Presión osmótica para Lactosa y D-Fructosa obtenidas experimentalmente y calculadas a partir de los modelos de Gibbs y Van't Hoff.

\begin{tabular}{|c|c|c|c|c|c|c|}
\hline \multirow{2}{*}{$\begin{array}{c}\text { Concentración } \\
(\% \mathrm{p} / \mathrm{v})\end{array}$} & \multicolumn{2}{|c|}{ Presión Osmótica Lactosa (bar) } & \multicolumn{3}{c|}{ Presión Osmótica D-Fructosa (bar) } \\
\cline { 2 - 7 } & Experimental & Van't Hoff & Gibbs & Experimental & Van't Hoff & Gibbs \\
\hline 0.5 & 3.55 & 0.37 & 0.92 & 2.68 & 0.70 & 1.75 \\
\hline 0.8 & 4.81 & 0.59 & 1.47 & 3.72 & 1.12 & 2.80 \\
\hline 1.0 & 2.97 & 0.74 & 1.84 & 4.35 & 1.40 & 3.50 \\
\hline 1.5 & 3.67 & 1.10 & 2.76 & 5.08 & 2.10 & 5.24 \\
\hline 2.0 & 4.16 & 1.47 & 3.68 & 3.89 & 2.80 & 6.98 \\
\hline 5.0 & 5.31 & 3.68 & 9.19 & 8.67 & 7.00 & 17.44 \\
\hline
\end{tabular}

En la Fig. 9 se comparan los valores obtenidos experimentalmente para la presión osmótica en función de la concentración molar de Lactosa y D-Fructosa. Para el rango de concentraciones estudiado, pueden realizarse dos observaciones interesantes: primero, que la presión osmótica es independiente de la naturaleza del azúcar y segundo, que existe un comportamiento lineal entre ambas variables, siendo ambos fenómenos concordantes con el modelo de Van't Hoff.

En general, se puede apreciar que para soluciones reales proveniente de riles de la industria azucarera, como mermeladas o jugos, donde los principales componentes son azúcares, como glucosa o D-Fructosa, los modelos de estimación de la presión osmótica no entregan predicciones adecuados. Por lo tanto, surge la necesidad de realizar pruebas de laboratorio para tener valores confiables y a partir de estos dimensionar el tamaño adecuado del equipo Osmosis Inversa que debe adquirirse para la industria. Otro punto importante que debe ser señalado, es que la energía requerida para operar un sistema de osmosis inversa, expresada como energía de presión (cuociente entre la presión subministrada al sistema y la densidad de la solución), dependerá de la presión osmótica de las soluciones, ya que este valor, indíca la mínima presión transmembrana con la cual es posible obtener un flux de permeado, tal cual lo señala el modelo de difusión-disolución (ecuación 1). Por lo tanto, la energía requerida para operar sistemas de osmosis inversa trabajando con soluciones ricas en azúcares, no es tan importante como en el caso de soluciones salinas, ya que estas siempre presentarán presiones osmóticas superiores, para una misma concentración en masa.

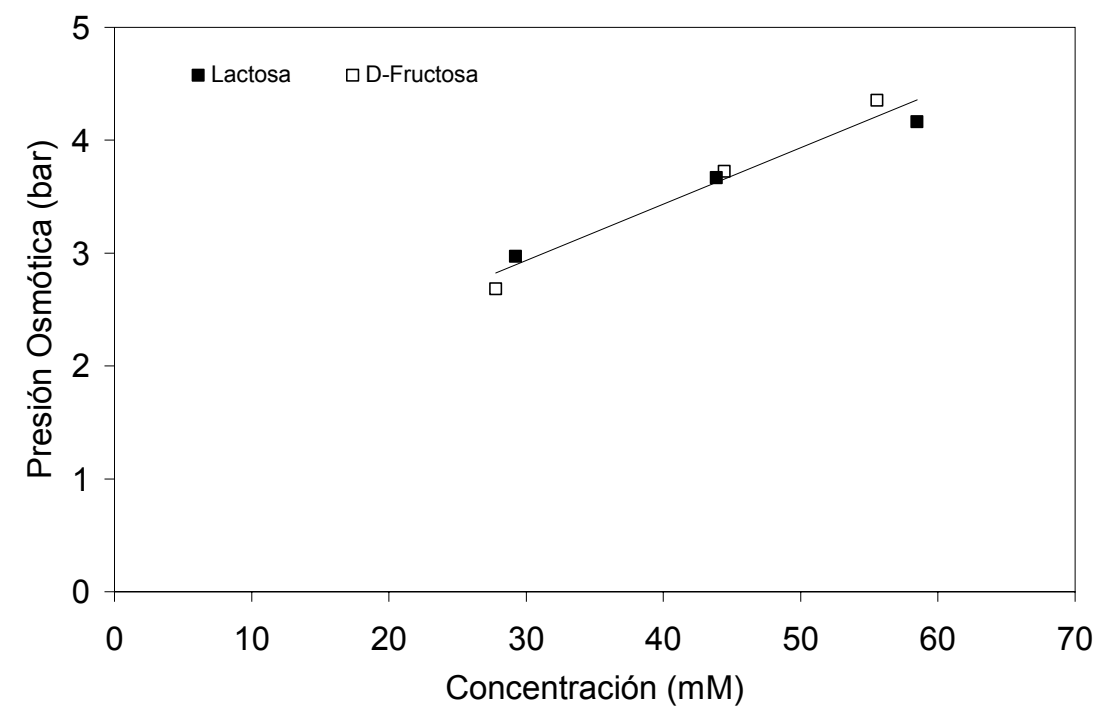

Fig. 8: Presión osmótica para Lactosa y D-fructosa, en función de la concentración molar.

Por otra parte, los efuentes líquidos generados por la industria láctea, durante el procesamiento de leches y yogures, son muy ricos en lactosa, por lo que su presión osmótica será consecuencia de la presencia de dicho azúcar. En cambio durante la producción de quesos, la presión osmótica será 
consecuencia de la presencia de las sales minerales que se encuentran en el suero, siendo estas principalmente $\mathrm{KCl}$ y $\mathrm{NaCl}$ (Cuartas et al., 2004), además de las solución de $\mathrm{NaCl}$ que se usan para la salazón de los quesos (Walstra et al., 1999).

En la práctica, para el primer caso (leche y yogurt), los valores de presión osmótica requeridos no son tan altos, ya que se encuentran en el rango de los obtenidos para lactosa al $5 \% \mathrm{p} / \mathrm{v}$, considerando que esta es la concentración que se puede encontrar en la leche y suero de yogurt (Walstra et al., 1999). Por el contrario, para el caso de las sales, ya se ha observado que existe un drástico aumento de la presión osmótica con el incremento de la concentración, tal cual se aprecia en las Figuras 2 y 3 . Esto genera consecuencias en la energía requerida para conseguir la separación. Por ejemplo, para el empleo de una bomba de 50 bar, pueden llegar a ocuparse 15 bar sólo por concepto de vencer la presión osmótica de la solución, cuando la concentración de $\mathrm{NaCl}$ es de un $2 \%$, lo que representa un alto requerimiento energético. A medida que la solución salina se concentra durante la operación del sistema, se incrementan aún más la presión osmótica. Por ello, en el momento de evaluar económicamente esta alternativa a nivel industrial, ya sea para la recuperación de azúcares, como para el reuso del agua, es preciso disponer de valores experimentales confiables para la presión osmótica de las soluciones, que permitan realizar las estimaciones adecuadas para determinar la máxima concentración que puede ser obtenida en el concentrado, de modo que el proceso sea económicamente rentable.

\section{CONCLUSIONES}

Fue posible determinar la presión osmótica para soluciones salinas y azucaradas utilizando el modelo de difusión-disolucion, en experiencias de osmosis inversa. Dicho modelo predice que la presión osmótica de una solución es igual a la presión transmembrana mínima requerida para obtener flux de permeado.

Al comparar los valores obtenidos a través de las curvas experimentales, se puede concluir que los modelos de Van't Hoff y de Gibbs no permiten realizar una buena estimación para las presiones osmóticas de soluciones salinas a concentraciones superiores al $2 \%$ de sales. Estos modelos sobre estiman el valor experimental. Asimismo, estos modelos no permiten obtener una estimación adecuada para el caso de Lactosa y D-fructosa, en un rango de concentración de un 0.5 a $5 \%$, pudiendo sobreestimar o subestimar la presión osmótica.

Por lo tanto, es preciso realizar experiencias de laboratorio que permitan la determinación de la presión osmótica de las soluciones, para poder implementar sistemas de osmosis inversa a nivel industrial y realizar un análisis confiable de los costos asociados a la operación de los sistemas. Este análisis debe hacerse solo basado en datos experimentales ya que los modelos de Van't Hoff, y Gibbs, propuestos para soluciones diluídas, no permiten obtener un cálculo preciso de la presión osmótica. La metodología propuesta en este trabajo puede ser utilizada para la determinación osmótica de cualquier soluto y a cualquier concentración, en sistemas de osmosis inversa.

\section{REFERENCIAS}

Balannec, B., M. Vourch, M. Rabiller-Baudry y B. Chaufer; Comparative Study of Different Nanofiltration and Reverse Osmosis Membranes for Dairy Effluent Treatment by Dead-end Filtration, Separ. Purif. Tech.: 42, 195-200 (2005).

Cheryan, M.; Ultrafiltration and Microfiltration Handbook. Second edition. 527p., CRS Press, Lancaster, U.S.A. (1998).

Cianci, F., L. Silva, L. Cabral, y M.V. Matta; Clarification and Concentration of Cashew Apple Juice by Membrane Processes, Ciênc. Tecnol. Aliment., ISSN: 0101-2061 (en línea), 25 (3), (2005). http://www.scielo.br/scielo.php?script=sci_arttext\&pid=S0101-20612005000300030\&lng=en\&nrm=iso Acceso: 26 de Julio (2008).

Cuartas, B., M.I. Alcaina y E. Soriano; Separation of Mineral Salts and Lactose Solutions Throught Nanofiltration Membranes, Food Sci. Tech. Int.: 10 (4), 255-262 (2004). 
Cuartas-Uribe, B., M.I. Alcaina-Miranda, E. Soriano-Costa, y A. Bes-Pía; Comparison of the Behavior of Two Nanofiltration Membranes for Sweet Whey Desmineralization, J. Dairy Sci.: 90, 1094-1101 (2007).

Čuda, P., P. Pospísil y J. Tenglerová; Reverse Osmosis in Water Treatment for Boilers, Desalination: 198, 41-46 (2006).

Fariñas, M.; Osmosis Inversa: Fundamentos, Tecnología y Aplicaciones, 225p., McGraw-Hill, Madrid, España (1999).

Galambos, I., y otros cuatro autores; High Organic Content Industrial Wastewater Treatment by Membrana Filtration, Desalination: 162, 117-120 (2004).

Gomes, E., E. Mendes, N. Pereira y S. Barros; Evaluation of the Acerola Juice Concentrated by Reverse Osmosis. Braz. Arch. Biol. Technol., ISSN: 1516-8913 (en línea), 48, 175-183 (2005). http://www.scielo.br/scielo.php?script=sci_arttext\&pid=S1516-89132005000400022\&lng=en\&nrm=iso Acceso: 26 de Julio (2008).

Hinková, A., Z. Bubník, P.Kadlec y J. Pridal; Potentials of Separation Membranes in the Sugar Industry, Separ. Purif. Tech.: 26, 101-110 (2002).

Hinková, A., y otros cuatro autores; Membrane Filtration in the Sugar Industry, Chem. Papers: $54\left(6^{a}\right)$, 375-382 (2000).

Hoek, E., A. Kim y M. Elimelech; Influence of Crossflow Filter Geometry and Shear Rate on Colloidal Fouling in Reverse Osmosis and Nanofiltration Separations, Environ. Eng. Sci.: 19 (6), 357-372 (2002).

Kessler, H.G.; Food and Bio Process Engineering: Dairy technology, Fifth edition, 694 p., Verlag A. Kessler, Munich, Germany (2002).

Ludwing, W., A. Seppälä y M. Lampinen; Experimental Study of the Osmotic Behavior of Reverse Osmosis Membranes for Different $\mathrm{NaCl}$ Solutions and Hydrostatic Pressure Differences, Exp. Thermal Fluid Sci.: 26, 963-969 (2002).

Madaeni, S., y S. Zereshki; Reverse Osmosis: an Energy Saving Option in Sugar Industry, Desalination: 200, 374-375 (2006).

Madaeni, S., y S. Zereshki; Reverse Osmosis Alternative: Energy Implication for Sugar Industry, Chem. Eng. Processing: 47, 1075-1080 (2008).

Manth T., J. Frenzel y A. van Vlerken; Large-Scale Application of UF and RO in the Production of Demineralized Water, Desalination: 118, 255-262 (1998).

Mirza S.; Reduction of Energy Consumption in Process Plants using Nanofiltration and Reverse Osmosis, Desalination: 224, 132-142 (2008).

Mulder, M.; Basic Principles of Membrane Technology, Second edition, 564p., Kluwer Academia Publisher, Dordrecht, Netherlands (1996).

Rektor, A., G. Vatai y E. Békássy-Molnár; Multi-step Membrane Process for the Concentration of Grape Juice, Desalination: 191, 446-453 (2006).

Silva F.T., J.G. Jardine y V.M. Matta, Orange Juice Concentration (Citrus sinensis) by Reverse Osmosis, Ciênc. Tecnol Aliment., ISSN: 0101-2061 (en línea), 18 (1), (1998). http://www.scielo.br/scielo.php?script=sci_arttext\&pid=S0101-20611998000100021\&lng=en\&nrm=iso. Acceso: 26 de Julio (2008).

Valentas, K., E. Rotstein y R. Sinch; Handbook of Food Engineering Practice, 718 p., CRS Press. Boca Raton, New York, U.S.A. (1997).

Vourch, M., B. Balannec, B. Chaufer y G. Dorange; Nanofiltration and Reverse Osmosis of Model Process Waters from the Dairy Industry to Produce Water for Use, Desalination 172; 245-256 (2005).

Walstra, P., y otros cuatro autores; Dairy Technology. Principles of Milk Properties and Processes, 727 p., Marcel Dekker Inc., New York, U.S.A. (1999). 